\title{
Molecular Dynamics simulations unveil the basis of the sequential binding of RsmE to the non-coding RNA RsmZ
}

Agustín Ormazábal, Juliana Palma* and Gustavo Pierdominici-Sottile**

Departamento de Ciencia y Tecnología, Universidad Nacional de Quilmes, Bernal, Buenos Aires, Argentina. Consejo Nacional de Investigaciones Científicas y Técnicas.

*Email: juliana@unq.edu.ar. Phone: +54 (11) 43657100 ext 5657

**Email: gsottile@unq.edu.ar. Phone: +54 (11) 43657100 ext 5657

List of figures presented in this section.

- Figure S1. Temporal evolution of the P-atom RMSDs from the initial structure, for representative simulations of each model.

- Figure S2. Nucleotides whose backbone dihedral angles were employed in the definition of the dPCA. Selected nucleotides are highlighted in yellow. The first nucleotide of the sequence is marked with light blue.

- Figure S3. Effect of changing the radius of the exclusion sphere on the relative accessibilities of alternative GGA BMs. Individual accessibilities are divided by the largest value obtained from all the simulations of L0 and R0, with all the putative exclusion radii.

- Figure S4. Projections of the trajectories onto the first two PC obtained from Cartesian PCA. Panel a) shows the results for the $\mathrm{L}$ conformer and b) for the $\mathrm{R}$ conformer. Data from different models are presented in different colors: blue for R0/L0, green for L1/R1, red for L2/R2 and yellow for L3/R3.

- Figure S5 Projections of the trajectories of the free froms of RsmZ onto the first two PC obtained from Cartesian PCA. Panel a) shows the results for L0 and b) for R0. Blue dots represent snapshots taken from the original simulations. Red dots represent snapshots taken from trajectories initiated after performing aMD.

- Figure S6 PDFs of the projections onto the first mode obtained by dPCA, for trajectories of L0 and R0 obtained after performing aMD.

- Figure S7. Tree structure network obtained from the density-clustering algorithm. The plot was done with the programs freely available at https:/github.com/lettis. 

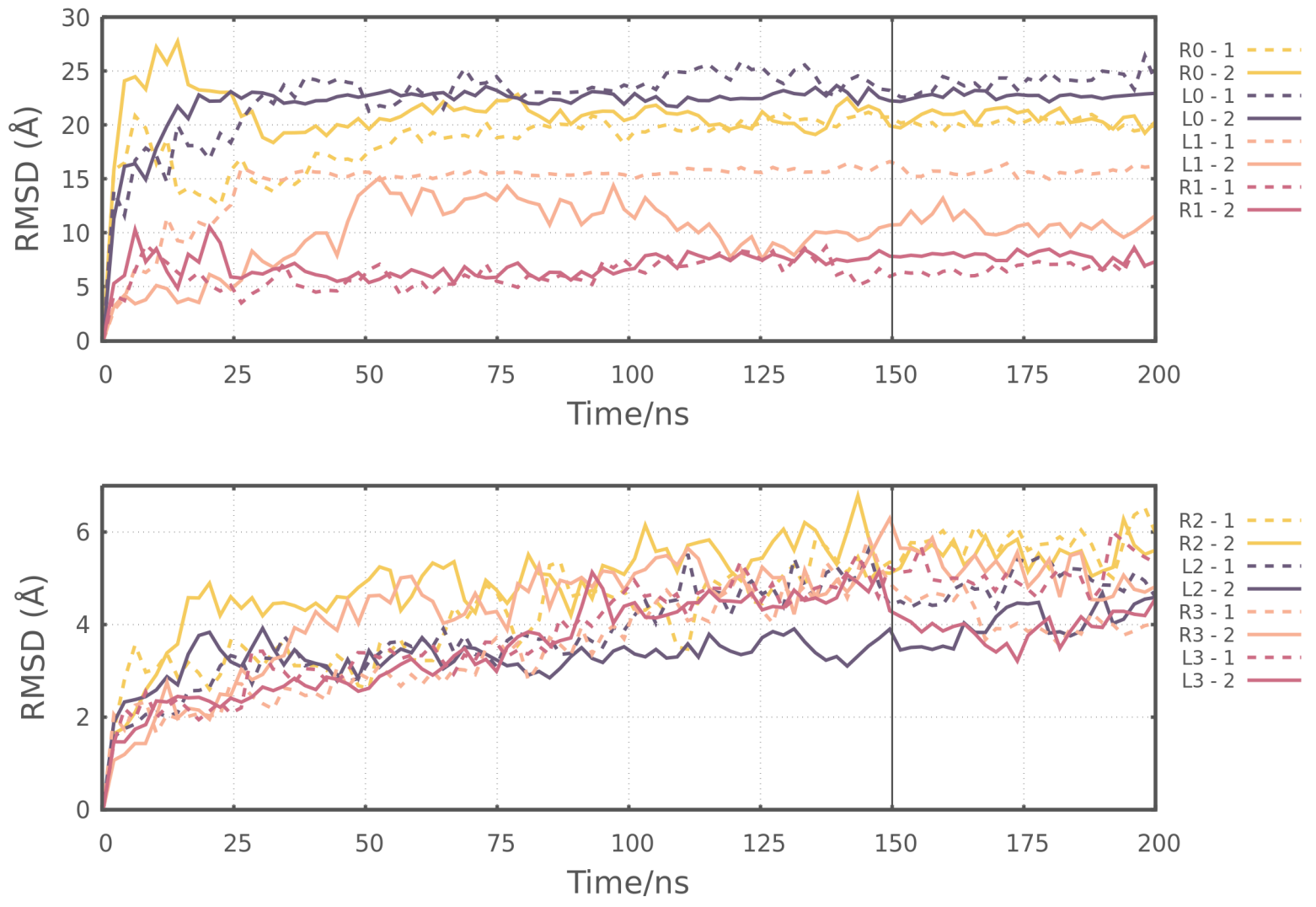

Figure S1: Temporal evolution of the P-atom RMSDs from the initial structure, for representative simulations of each model. 


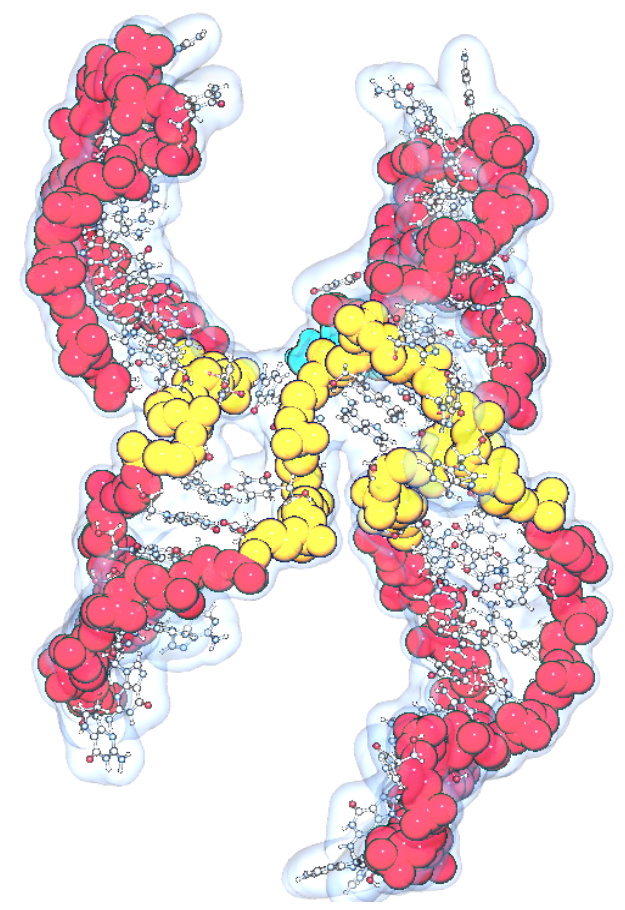

Figure S2: Nucleotides whose backbone dihedral angles were employed in the definition of the dPCA. Selected nucleotides are highlighted in yellow. The first nucleotide of the sequence is marked with light blue. 


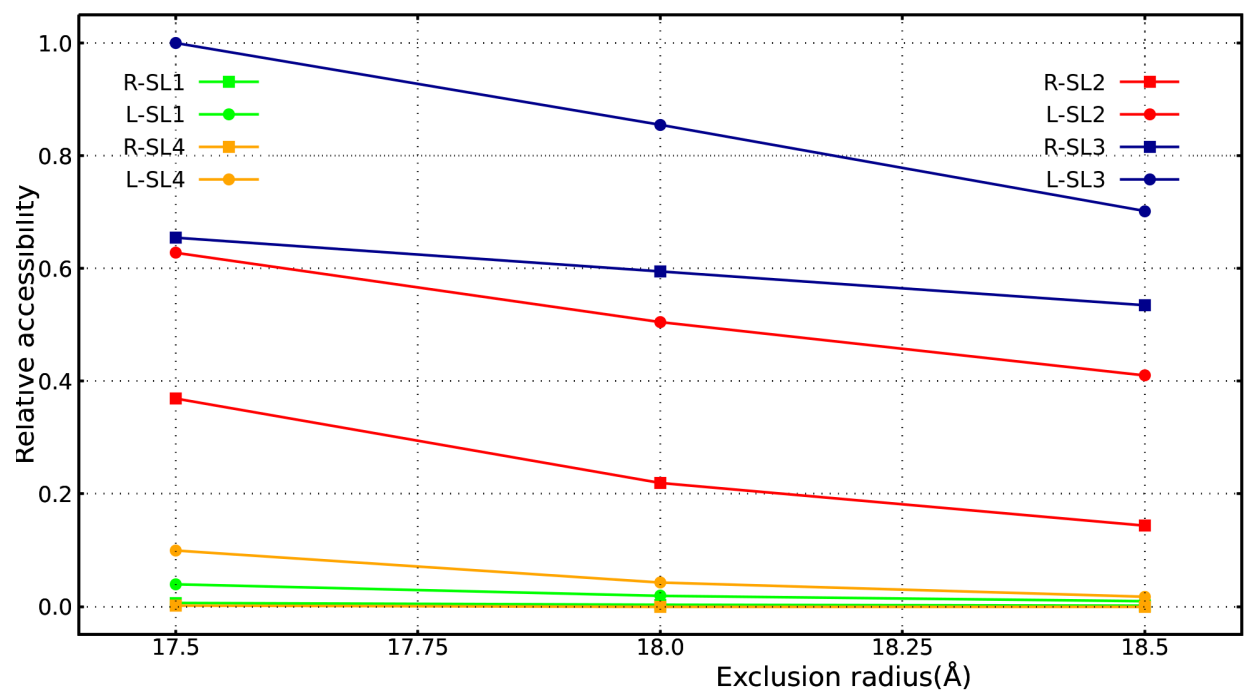

Figure S3: Effect of changing the radius of the exclusion sphere on the relative accessibilities of alternative GGA BMs. Individual accessibilities are divided by the largest value obtained from all the simulations of L0 and R0, with all the putative exclusion radii. 

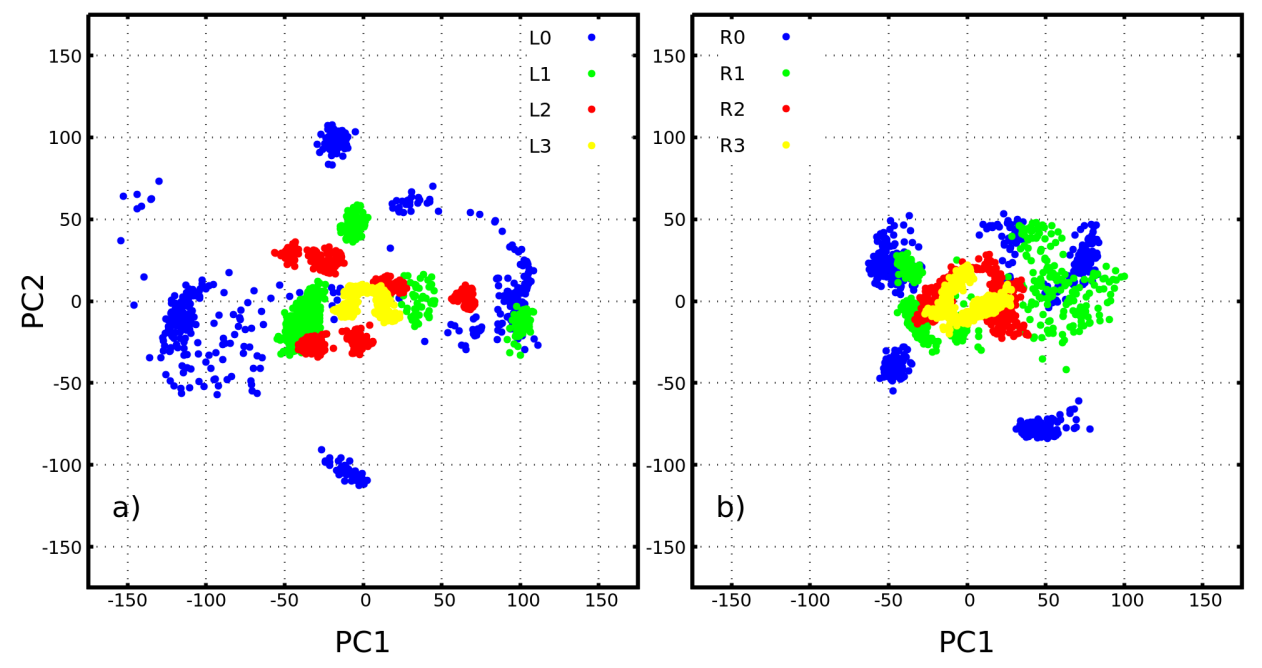

Figure S4: Projections of the trajectories onto the first two PC obtained from Cartesian PCA. Panel a) shows the results for the $\mathrm{L}$ conformer and b) for the $\mathrm{R}$ conformer. Data from different models are presented in different colors: blue for R0/L0, green for L1/R1, red for L2/R2 and yellow for L3/R3. 

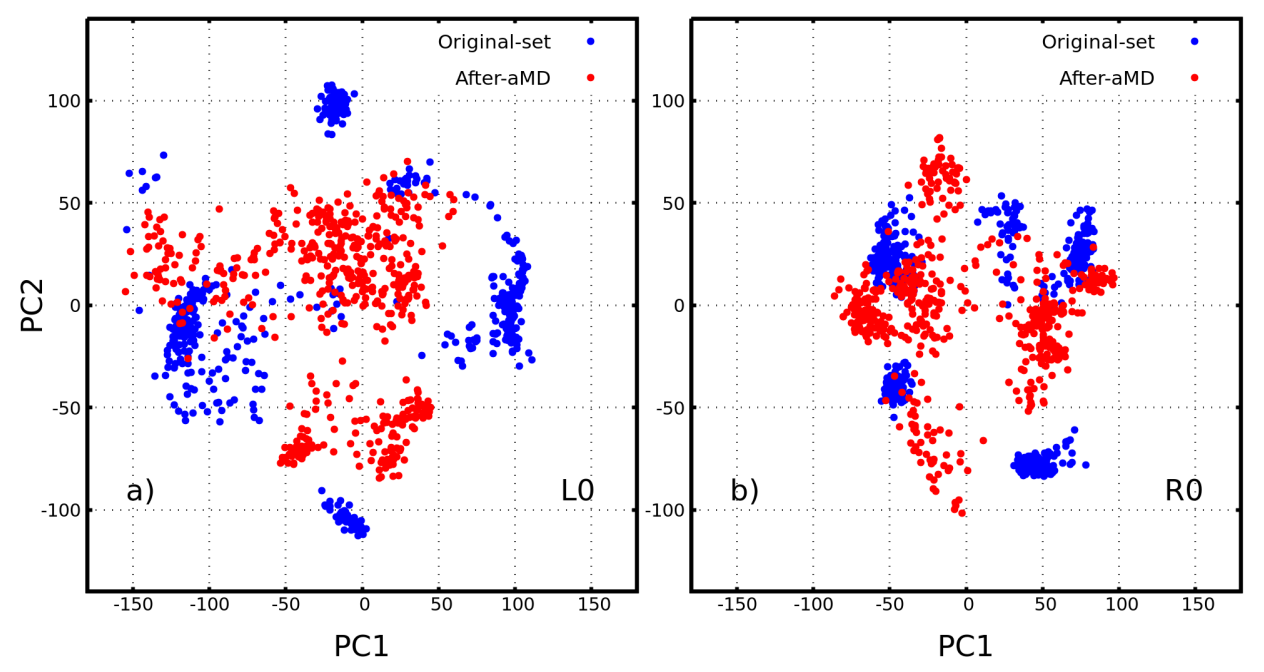

Figure S5: Projections of the trajectories of the free forms of RsmZ onto the first two PC obtained from Cartesian PCA. Panel a) shows the results for L0 and b) for R0. Snapshots obtained from the original simulations are shown in blue. Snapshots taken from trajectories initiated after practicing aMD are shown in red. 


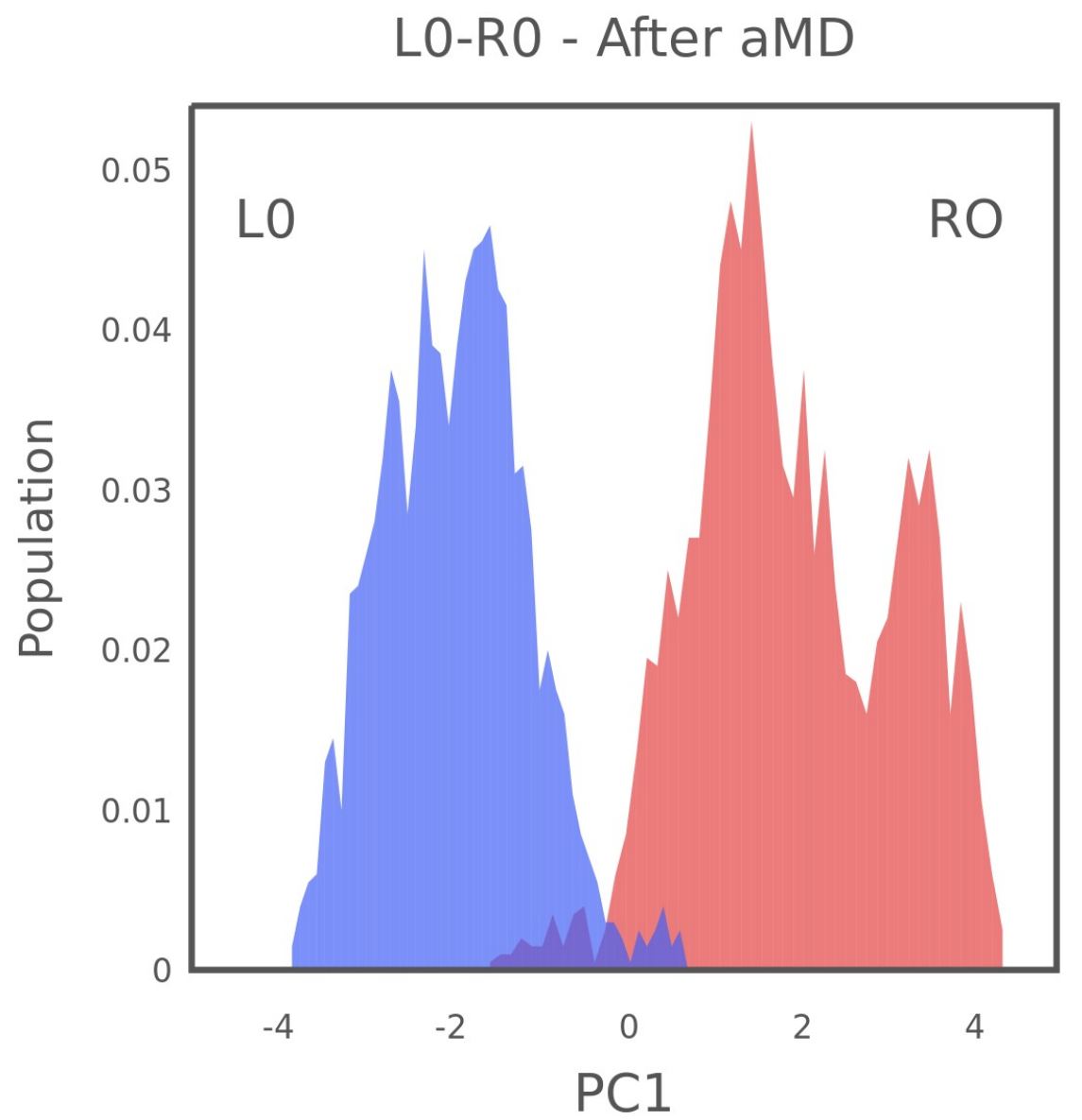

Figure S6: Probability density functions for the projections of the trajectories of L0 and R0 onto the first principal component obtained by dPCA The analysis were performed with the snapshots obtained after performing aMD. Data corresponding to the trajectories of the $\mathrm{L}$ conformer are presented in blue while those corresponding to the $\mathrm{R}$ conformer are presented in red. 

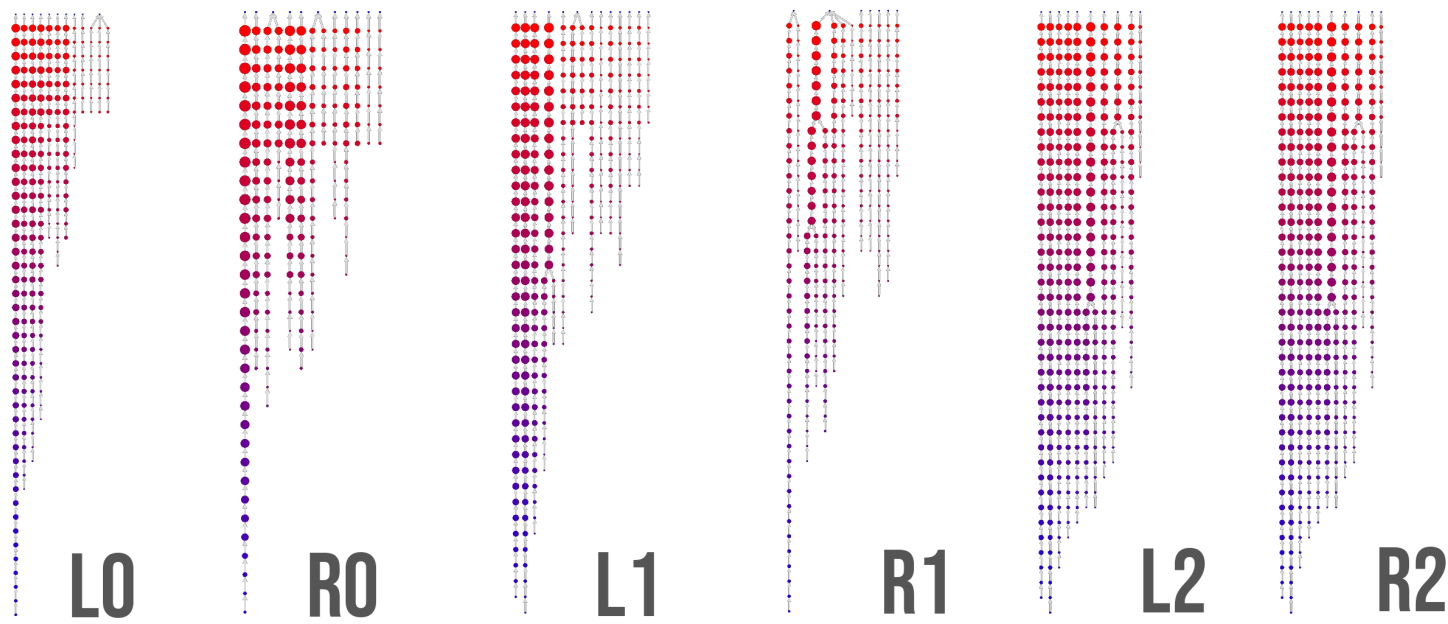

Figure S7: Tree structure network obtained from the density-clustering algorithm. The plot was done with the programs freely available at https://github.com/lettis (see references 71 and 72 of the main text). 